\title{
Dornbach, Stefan
}

\section{Zeitmanagement in der beruflichen Bildung}

formal überarbeitete Version der Originalveröffentlichung in:

formally revised edition of the original source in:

Die berufsbildende Schule 66 (2014) 11/12, S. 368-372

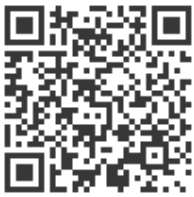

Bitte verwenden Sie in der Quellenangabe folgende URN oder DOI /

Please use the following URN or DOI for reference:

urn:nbn:de:0111-pedocs-109201

10.25656/01:10920

https://nbn-resolving.org/urn:nbn:de:0111-pedocs-109201

https://doi.org/10.25656/01:10920

\section{Nutzungsbedingungen}

Gewährt wird ein nicht exklusives, nicht übertragbares, persönliches und beschränktes Recht auf Nutzung dieses Dokuments. Dieses Dokument is ausschließlich für den persönlichen, nicht-kommerziellen Gebrauch bestimmt. Die Nutzung stellt keine Übertragung des Eigentumsrechts an diesem Dokument dar und gilt vorbehaltlich der folgenden Einschränkungen: Auf sämtlichen Kopien dieses Dokuments müssen alle Urheberrechtshinweise und sonstigen Hinweise auf gesetzlichen Schutz beibehalten werden. Sie dürfen dieses Dokument nicht in irgendeiner Weise abändern, noch dürfen Sie dieses Dokument für öffentliche oder kommerzielle Zwecke vervielfältigen, öffentlich ausstellen, aufführen, vertreiben oder anderweitig nutzen.

Mit der Verwendung dieses Dokuments erkennen Sie die Nutzungsbedingungen an.

\section{Terms of use}

We grant a non-exclusive, non-transferable, individual and limited right to using this document

This document is solely intended for your personal, non-commercial use. Use of this document does not include any transfer of property rights and it is conditional to the following limitations: All of the copies of this documents must retain all copyright information and other information regarding legal protection. You are not allowed to alter this document in any way, to copy it for public or commercial purposes, to exhibit the document in public, to perform, distribute or otherwise use the document in public.

By using this particular document, you accept the above-stated conditions of use.

\section{Kontakt / Contact:}

\section{peDOCS}

DIPF | Leibniz-Institut für Bildungsforschung und Bildungsinformation Informationszentrum (IZ) Bildung

E-Mail: pedocs@dipf.de

Internet: www.pedocs.de

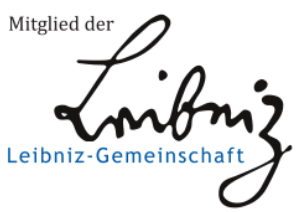




\section{Stefan Dornbach}

\section{Zeitmanagement in der beruflichen Bildung}

Die berufsbildende Schule (BbSch) 66 (2014) 11/12, S. 368-372

Die moderne Arbeitswelt verlangt von ihren Arbeitnehmern ausgeprägte Fähigkeiten der zeitlichen Selbstorganisation. Dies gilt zunehmend auch für Tätigkeiten mit geringen Anforderungen an die fachliche Qualifikation. Teilnehmer an Maßnahmen zur Berufsvorbereitung müssen häufig mit einer Helfertätigkeit in das Berufsleben einsteigen. Sind sie auf die dortigen zeitlichen Anforderungen vorbereitet und wie kann das Bildungssystem dazu beitragen, entsprechende Fähigkeiten zu entwickeln?

Zeit kann man nicht managen. Diesen Satz höre ich oft, wenn ich mein Forschungsthema vorstelle. Und wörtlich betrachtet ist er richtig. Wie immer man „Zeit" auch versteht - sie entzieht sich individueller Manipulation. Letztlich ist Zeit ein gesellschaftliches Konstrukt. Das jeweils vorherrschende Verständnis von Zeit passt sich den gegebenen sozialen Rahmenbedingungen an und wirkt dabei selbst normbildend.

In modernen Gesellschaften haben Arbeitnehmer eine Vielzahl zeitlicher Anforderungen zu erfüllen, die es in dieser Form vor wenigen Jahrzehnten noch nicht gab. Für den „einfachen“ Arbeiter in der tayloristischen Fabrik oder den unteren Angestellten in der preußisch geprägten Staatsbürokratie reichte es bis dahin aus, pünktlich am Arbeitsplatz zu erscheinen und sich den dort vorgegebenen zeitlichen Rhythmen zu unterwerfen. So war "Zeitmanagement“ bei seinem Aufkommen als Modebegriff in den 80er-Jahren des 20. Jahrhunderts noch höheren Angestellten vorbehalten, eben denen, welche die Zeit der anderen „managten“. Dabei gerieten sie oftmals selbst in Überforderungssituationen, aus denen sie sich mit persönlichem Zeitmanagement zu befreien suchten. Die Verheißung dieser Möglichkeit schuf einen neuen Markt an Seminaren und eine Bibliothek an Ratgeberliteratur: Der Katalog der Deutschen Nationalbibliothek verzeichnet mehr als 300 Monografien, die den Begriff "Zeitmanagement“ im Titel tragen und fast ausschließlich mit einem „Gewinn“ an Zeit durch die Befolgung dort beschriebener Strategien der Arbeitsgestaltung werben.

Inzwischen ist das Problem der komplexen zeitlichen Normen und der damit einhergehenden Gefahr der Überforderung auch in den „einfachen“ beruflichen Tätigkeiten angekommen. Während diese im deutschen Industriesektor durch die nahezu restlose Automatisierung kontinuierlich abnehmen, schafft der Dienstleistungsmarkt neue Beschäftigungen, für deren Ausübung ein geringes fachliches Qualifikationsniveau ausreicht. Diese sind allerdings oft mit hohen zeitlichen Anforderungen verbunden. Durch für den Arbeitgeber flexible Arbeitszeitmodelle müssen die Arbeitnehmer in diesem Bereich zur selbstständigen zeitlichen Organisation ihres Arbeitsalltages unter ständig wechselnden Bedingungen in der Lage sein, was Einsatzzeiten und -orte betrifft. Durch die Ausdehnung der Arbeitszeiten in den früheren Freizeitbereich wird auch ihre Abstimmung mit privaten 
zeitlichen Anforderungen immer anspruchsvoller. Hinzu kommt die zeitliche Koordination von einzelnen Arbeitsschritten, die im Dienstleistungsbereich vielen Störfaktoren unterliegt, also eine ständige zeitliche Um- und Neuplanung erfordert. Arbeitnehmer in Hilfstätigkeiten werden dadurch immer häufiger Manager ihrer eigenen Arbeitszeit.

Daraus ergibt sich die Frage, an welchen Stellen in unserem Bildungssystem entsprechende Fähigkeiten vermittelt werden und wie das Lernen in dieser Hinsicht gefördert werden kann.

In der Studie „Zeitmanagement in der beruflichen Bildung“ wurde deshalb der Versuch unternommen, den Begriff des Zeitmanagements für die Bildungsforschung nutzbar zu machen (Dornbach 2014). Überlegungen, den seit einiger Zeit in der Ratgeberliteratur verwendeten Begriff „Selbstmanagement“ zu benutzen, wurden schnell verworfen, schließlich ist das ganze menschliche Leben spätestens ab dem Erwachsenwerden so etwas wie "Selbstmanagement“. Um den Begriff des Zeitmanagements wissenschaftlich zu schärfen, fragte ich zunächst nach der subjektiven Bedeutung der Zeit und ihres Einflusses bei der Eröffnung und Entwicklung von Handlungsperspektiven. Wie tragfähig ist das vorherrschende mechanisch-physikalische Zeitverständnis? Schon bei der Organisation der Fabrikarbeit stand ein physikalisches Zeitmodell im Vordergrund: Zeit wurde als eine in beliebige Einheiten teilbare Größe betrachtet, die dazu diente, Produktionsprozesse zu optimieren. Im Zentrum dieser Entwicklung stand die effektive Zeitausnutzung, die Ökonomie der Zeit. Diese Zeitökonomie wurde ein wichtiger Motor für die kapitalistische Entwicklung. Sie eröffnete die Chance, in stetig kürzer werdenden Zeitspannen immer größere Mengen an Produkten herzustellen. Auch die zeitlichen Rhythmen in der deutschen Schule ähneln noch oft den Abläufen in der tayloristischen Fabrik. Das Klingelzeichen ersetzt die Sirene beim Anzeigen von Beginn-, Pausen- und Endzeiten. Dabei wird der Schultag in 45-MinutenEinheiten eingeteilt, an deren Ende jeweils ein bestimmtes Teilwissen beim Schüler als Produkt entstanden sein soll.

Dem gegenüber steht die beschriebene Notwendigkeit zum Erlernen zeitlicher Selbstorganisation. Die Fähigkeit zu selbstständigem Lernen und Handeln ist ein deklariertes Ziel der dualen Berufsausbildung. Die Anwendung dieser Fähigkeit in modernen Produktions- und Dienstleistungskonzepten erfordert vom tätigen Individuum weitaus mehr als ein an vorgegebene Rhythmen angepasstes zeitliches Agieren. Vielmehr zeichnet sich ab, dass Techniken des Zeitmanagements eine Schlüsselrolle in selbstregulierten Lern- und Arbeitsprozessen einnehmen können.

Individuelles Zeitmanagement ist aber nicht allein eine „Technik“, sondern abhängig von lebensweltlichen Erfahrungen, daraus entstehenden Selbstkonzepten und Motivationslagen. Das „Erwartung-mal-Wert-Modell“ erscheint daher als geeignet, um die subjektiven Lebenssituationen und Motivationslagen des tätigen Individuums besser zu verstehen. Dieses Modell geht davon aus, dass die Motivation, bestimmte Handlungen durchzuführen, sich aus dem Produkt aus der Erwartung an das Ergebnis der Handlung und des individuellen Werts, welcher dem Ergebnis zugemessen wird, ergibt $\left(M=E{ }^{*} W\right)$. Der individuelle Wert einer ZeitmanagementAktivität wird wie das Zeiterleben durch kulturelle Voraussetzungen und Lebensentwürfe mitbestimmt. Die Erwartung an das Ergebnis einer zeitbezogenen Handlung ergibt sich unter anderem aus Erfahrungen mit Erfolg und Misserfolg. Damit wird „Zeitmanagement“ nicht nur als „Zeitökonomie“ verstanden, sondern als die Fähigkeit, Handlungsentwürfe und -pläne in einer zeitlichen Dimension zu strukturieren und zu realisieren. 
Wie aber erfahren Individuen Zeit? Zeiterleben und zeitliche Wertvorstellungen bilden sich in verschiedenen Stufen der Persönlichkeitsentwicklung unterschiedlich heraus. Betrachtet man es im Kontext von Handlungsentwürfen und Handlungsmöglichkeiten, so wird deutlich, dass Handlungssituationen mit geringen Chancen zur Selbstregulation Ansätze des Zeitmanagements beschädigen oder zerstören können.

Mit Alessandro Cavalli lassen sich fremd- und eigengesteuerte Typen des individuellen Umgangs mit Zeit bei Jugendlichen unterscheiden (Cavalli 1988). Junge Menschen, die wenig elterliches Unterstützungsverhalten und ein schwaches soziales Umfeld erfahren haben, sind häufig fremdstrukturierte Typen. Gerade bei diesen Idealtypen können fehlende Zukunftsperspektiven destruktive Wirkungen auf die Motivation zur Ausübung von Zeitmanagement haben. In unserer Studie unterstellten wir, dass ein großer Teil der Teilnehmer an Maßnahmen zur Berufsvorbereitung zu dieser Risikogruppe für die individuelle ZeitmanagementEntwicklung gehörte. Mit dem Erwartung-mal-Wert-Modell lässt sich das Problem idealtypisch erklären. Die sozialen Bedingungen und die Sozialisationsmilieus leisten einen Beitrag zur Entwicklung einer entsprechenden Zeitmanagementstrategie. Jugendliche aus einem schwach strukturierten sozialen Umfeld erlernen deshalb vorwiegend, auf fremdbestimmte Strategien zum Zeitmanagement zurückzugreifen. Zeitliche Selbstbestimmung stellt für sie einen geringen Wert dar. Perspektivlosigkeit und unwirtschaftlicher Umgang mit ihrer Lebenszeit - etwa durch Warteschleifen multiplizieren dieses Problem: Nach dem Erwartung-mal-Wert-Modell wird in diesem Fall die Motivation, Zeitmanagement aktiv auszuüben, durch die Absenkung beider Faktoren des Produkts erheblich gemindert.

Aufbauend auf diesen Vorüberlegungen wurde eine empirische Studie konzipiert. In der quantitativen Hauptstudie fand eine Fragebogenerhebung statt, in welcher bei Teilnehmern an Maßnahmen zur Berufsausbildung und bei Auszubildenden Selbsteinschätzungen zum Zeitmanagementverhalten in konstruierten Situationen abgefragt wurden.

\section{Die konstruierten Situationen}

Die Party

Stellen Sie sich Folgendes vor: In etwa zwei Stunden wollen Sie sich mit Ihren Freunden zu einer Party treffen. Sie haben zugesagt, einen großen Salat mitzubringen. Sie schneiden deshalb Gemüse, würden aber lieber etwas anderes machen. Sie verlieren die Lust daran, den Salat zuzubereiten. Was tun Sie?

Die Schulaufgabe

Stellen Sie sich Folgendes vor: Sie arbeiten zu Hause an einer Aufgabe, die Sie unbedingt bis morgen für die Berufsschule erledigen müssen. Dafür haben Sie noch zwei Stunden Zeit. Sie würden aber lieber etwas anderes machen und verlieren die Lust an der Schulaufgabe. Was tun Sie?

Die Bewerbungsmappe

Stellen Sie sich Folgendes vor: Heute Nachmittag haben Sie ein Vorstellungsgespräch für einen Ausbildungsplatz, den Sie unbedingt haben möchten. Sie wollen eine besonders gute Bewerbungsmappe zum Gespräch mitbringen. Sie schreiben deshalb gerade den Lebenslauf neu. Sie würden aber lieber etwas anderes machen und verlieren die Lust an der Bewerbungsmappe. Was tun Sie? 
Zur Vorbereitung dieser Hauptstudie diente eine qualitative Vorstudie. Mittels Leitfadeninterviews erfragte ich individuelle Handlungsmuster der Befragten, die sich später mittels der Bildung von Idealtypen, welche jeweils bestimmte Zeitmanagement-Muster verkörperten, quantitativ einordnen ließen. Auf dem Hintergrund dieser Typen wurden Handlungs- und Entscheidungssituationen mit vorgegebenen Handlungsalternativen als Antwortmöglichkeiten konstruiert.

Die Ausgestaltung der Situationen orientierte sich an der Volitionsforschung und Erkenntnissen zum Zeitmanagement aus der psychologischen Forschung (u. a. König und Kleinmann 2004). Volitionale Strategien können demnach ein bewusst einsetzbares Mittel zur zeitlichen Selbstbestimmung sein, somit stellen ihre Beherrschung oder auch ihr unbewusster Einsatz entscheidende Komponenten des individuellen Zeitmanagements dar. Für die qualitative Studie wurde ein Interviewleitfaden erstellt, der neben den konstruierten Situationen einen soziobiografischen Teil beinhaltete.

Mithilfe der soziobiografischen Daten war es möglich, verschiedene Hypothesen zu prüfen. Einerseits wird in der bildungspolitischen Diskussion oft behauptet, dass mangelnde soziale Eingebundenheit und Arbeitslosigkeit nicht nur die zeitlichen Rhythmen der unmittelbar Betroffenen zerstören, sondern diese destruktiven Strukturen sich auch auf folgende Generationen übertragen. Um diese Annahme zu überprüfen, wurden die familiäre Situation und die Berufstätigkeit der Eltern erfasst.

So wie die Erwerbstätigkeit prägt auch ein Migrationshintergrund familiäre Verhaltensweisen. Es ist denkbar, dass verschiedene, durch einen Migrationshintergrund geprägte familiäre Kulturen zu verschiedenen Zeitmanagement-Verhaltensweisen bei den Kindern führen. Auch diese Hypothese sollte mithilfe der soziobiografischen Daten in der qualitativen Studie überprüfbar gemacht werden.

Es wurden acht Auswertungskategorien festgelegt, die idealtypisch individuelle Verhaltensweisen zusammenfassten, die als Reaktionen auf die konstruierten Situationen denkbar waren:

1. Volition (Einsatz von Willenskraft, der von verinnerlichten Einstellungen oder Selbstzwang gesteuert sein kann),

2. Prokrastination (Verschieben der Handlungsabsicht auf eine bestimmte oder unbestimmte Zeit),

3. Präferenzwechsel (Abbruch der ursprünglichen Handlungsabsicht zugunsten einer kurzfristig attraktiveren Alternative),

4. Multitasking (gleichzeitiges Erledigen der Handlungsabsicht und der unerwartet auftretenden Alternative),

5. Delegation (Abgeben der Aufgabe an jemand anderen),

6. Präventives Vermeiden (im Vorfeld organisiertes Abschirmen der Handlungsabsicht gegen Alternativen),

7. Hopping (Hin- und Herspringen zwischen Handlungsabsicht und Alternative),

8. Vergessen/Verdrängen (unbewusstes Aufgeben der Handlungsabsicht).

In der Auswertung der Interviews ließ sich feststellen, dass die nach den theoretischen Überlegungen Erfolg versprechendste Strategie - die des präventiven 
Vermeidens von Störungen der Handlungsabsicht - kaum angewendet wurde. Das Gleiche galt für das Hopping, die Delegation und das Vergessen/Verdrängen. Etwas häufiger wurde das Multitasking gebraucht, vor allem bei den weiblichen Befragten.

Insgesamt reagierten die Interviewten auf die konstruierten Situationen schwerpunktmäßig mit Volition und Prokrastination. Bei zeitlich nicht klar begrenztem Verschieben entstand häufig die Gefahr eines Präferenzwechsels. In der qualitativen Inhaltsanalyse der Interviews fiel unmittelbar auf, dass vor allem die weiblichen Befragten mit volitionalen Strategien arbeiteten und sie überwiegend als Selbstverständlichkeit einsetzten, ohne größere bewusste Willensanstrengungen ausüben zu müssen. Wenn die männlichen Befragten volitionale Strategien anwandten, dann waren ihre dabei entstehenden individuellen Kosten in Bezug auf den Verbrauch motivationaler Ressourcen deutlich höher, weil die Strategien nicht selbstverständlich „im Hintergrund arbeiteten“, sondern immer wieder als bewusster Selbstzwang verstärkt werden mussten.

Die männlichen Befragten bewältigten die konstruierten Situationen häufiger mithilfe von Prokrastination als die weiblichen. Dieses Verschieben der ursprünglichen Handlungsabsicht war dabei meist stärker risikobehaftet als bei der Anwendung dieser Strategie durch die weiblichen Befragten. Das lag daran, dass die männlichen Befragten sich deutlich seltener einen konkreten Zeitpunkt für die Wiederaufnahme der ursprünglichen Handlung setzten.

Die konstruierten Situationen Die Party Stellen Sie sich Folgendes vor: In etwa zwei Stunden wollen Sie sich mit Ihren Freunden zu einer Party treffen. Sie haben zugesagt, einen großen Salat mitzubringen. Sie schneiden deshalb Gemüse, würden aber lieber etwas anderes machen. Sie verlieren die Lust daran, den Salat zuzubereiten. Was tun Sie? Die Schulaufgabe Stellen Sie sich Folgendes vor: Sie arbeiten zu Hause an einer Aufgabe, die Sie unbedingt bis morgen für die Berufsschule erledigen müssen. Dafür haben Sie noch zwei Stunden Zeit. Sie würden aber lieber etwas anderes machen und verlieren die Lust an der Schulaufgabe. Was tun Sie? Die Bewerbungsmappe Stellen Sie sich Folgendes vor: Heute Nachmittag haben Sie ein Vorstellungsgespräch für einen Ausbildungsplatz, den Sie unbedingt haben möchten. Sie wollen eine besonders gute Bewerbungsmappe zum Gespräch mitbringen. Sie schreiben deshalb gerade den Lebenslauf neu. Sie würden aber lieber etwas anderes machen und verlieren die Lust an der Bewerbungsmappe. Was tun Sie?

Diese in der qualitativen Vorstudie mit einer Stichprobe von 24 Interviews zutage getretenen Zusammenhänge wurden nun in der Hauptuntersuchung quantitativ überprüft. Dazu wurde ein Fragebogen konstruiert, der drei Situationen enthielt. Diese gaben Herausforderungen an das Zeitmanagement in verschiedenen Sphären wider, die sich unter den Begriffen „Schule“, „Privates“ und „,berufliche Entwicklung“ kategorisieren ließen. Für das Widerspiegeln der schulischen Sphäre wurde das Erledigen einer häuslichen Schulaufgabe ausgewählt, weil in einer solchen Situation Zeitmanagement-Fähigkeiten deutlich stärker zum Tragen kommen als in direkt schulischen Situationen, deren zeitliche Rahmenbedingungen meist relativ starr vorgegeben sind. Für die Sphäre des Privaten wurde die Vorbereitung einer Party, für die der beruflichen Entwicklung die Anfertigung einer Bewerbungsmappe gewählt.

Der Fragebogen wurde von 243 Teilnehmern an Maßnahmen zur Berufsvorbereitung ausgefüllt. Die zusätzlichen 114 Befragten der Vergleichsgruppe befanden sich in Ausbildung und hatten damit bereits eine höhere berufliche Qualifikationsstufe erreicht. So sollte überprüft werden, ob es Anzeichen gab, dass Zeitmanagement 
eine sich in diesem Qualifikationsprozess weiterentwickelnde Fähigkeit im Sinne einer erlernbaren Technik darstellte.

$\mathrm{Zu}$ jeder der drei konstruierten Situationen wurden abgeleitet von den Ergebnissen und Erfahrungen der qualitativen Vorstudie neun Items erstellt, in denen die folgenden Handlungstendenzen operationalisiert waren:

a. Präferenzwechsel mit völligem Abbruch der Handlungsabsicht,

b. Präferenzwechsel,

c. zeitlich unbestimmte Prokrastination,

d. zeitlich bestimmte Prokrastination,

e. V olitionsanstrengung durch Selbstzwang,

f. Delegation,

g. präventives Vermeiden der Erschöpfung der volitionalen Ressourcen,

h. Notwendigkeit zur Motivation von außen,

i. Volitionssteuerung durch verinnerlichte Einstellungen.

$\mathrm{Zu}$ jeder dieser Handlungsmöglichkeiten wurde eine vierstufige Auswahlskala von „trifft zu“ bis „trifft nicht zu“ vorgegeben. Die Fragebögen wurden mithilfe der Faktorenanalyse ausgewertet. Was den Einfluss des Geschlechts betrifft, so bestätigten sich in diesem Verfahren die Ergebnisse der qualitativen Vorstudie. Die männlichen Befragten neigten eher zu den risikobehafteten Vermeidungsstrategien, während die weiblichen Befragten deutlich stärker mit volitionalen Strategien arbeiteten. Diese deutlichen geschlechtsspezifischen Unterschiede im Zeitmanagementverhalten ließen sich als Ausdruck unterschiedlicher Selbstkonzepte interpretieren, die auch zu verschiedenen Wertzuschreibungen und Erwartungen an die eigenen Handlungen führten. Nach dem Erwartung-mal-Wert-Modell bildeten sich dadurch unterschiedliche motivationale Lagen, die als entscheidender Einflussfaktor auf das Zeitmanagement fungierten.

Ähnliches galt für den Einfluss des Migrationshintergrundes der Befragten. Auch hier ließen sich die aufgetretenen Unterschiede im Zeitmanagement durch verschiedene Selbstkonzepte und Zeiterleben erklären, die unterschiedliche Werte und Erwartungen an die Handlungen begründeten. Die Befragten mit Migrationshintergrund wendeten Vermeidungsstrategien erkennbar anders an als die Befragten ohne einen solchen Hintergrund. In der Situation „Party“ nutzen sie deutlich seltener, in der Situation „Bewerbungsmappe“ deutlich häufiger Vermeidungsstrategien. Die Situation „Party“ entsprach aber nicht der Erfahrungswelt der Jugendlichen mit Migrationshintergrund. Vielen der weiblichen Befragten waren Partys von ihren Eltern verboten, viele der männlichen Jugendlichen meldeten zurück, dass sie niemals die Aufgabe der Zubereitung eines Salats für eine Party übernehmen würden. Die Antworten waren also häufig nicht durch reales Zeiterleben geprägt. Deshalb ist davon auszugehen, dass die Jugendlichen mit Migrationshintergrund eher zu Vermeidungsstrategien neigten, so wie es sich in der Situation „Bewerbungsmappe“ zeigte. Außerdem arbeiteten sie insgesamt stärker mit volitionalen Strategien.

Da sich die Auszubildenden in der Vergleichsgruppe zum Großteil am Beginn ihres Ausbildungsganges befanden, war auszuschließen, dass sie sich bereits in großem Umfang Techniken des Zeitmanagements in Lehr-Lern-Zusammenhängen angeeignet hatten, die den Teilnehmern der Maßnahmen zur Berufsvorbereitung 
nicht zur Verfügung gestanden hatten. Es konnte deshalb davon ausgegangen werden, dass bereits der Übergang in den Status eines Auszubildenden ein verändertes Selbstkonzept und neue motivationale Lagen hervorrief, welche Auswirkungen auf das Zeitmanagement hatten.

Bei der Betrachtung der Berufstätigkeit der Eltern wurde deutlich, dass das Vorhandensein einer solchen Tätigkeit zwar das Zeitmanagement-Verhalten der Kinder beeinflusste, dies aber nicht im Sinne eines negativen Einflusses von Arbeitslosigkeit des Vaters auf die nächste Generation durch die Zerstörung zeitlicher Strukturen in der Familie zu werten war. Vielmehr zeigte sich, dass das Vorhandensein oder Nicht-Vorhandensein einer Berufstätigkeit des Vaters unterschiedliche berufliche Selbstkonzepte bei den Kindern erzeugte, die sich auf deren motivationale Lagen und damit inr Zeitmanagement auswirkten. Diese konnten sich sowohl in Abgrenzung zur Berufstätigkeit oder Nicht-Berufstätigkeit des Vaters zeigen, als auch im damit Konformgehen, was das eigene Zeiterleben und die damit zusammenhängenden Wertvorstellungen betraf.

In der empirischen Studie zeigte sich insgesamt am deutlichsten, dass männliche und weibliche Teilnehmer an Maßnahmen zur Berufsvorbereitung unterschiedliche Zeitmanagement-Strategien anwendeten. Man könnte deshalb anhand der Ergebnisse von einem „weiblichen“ und einem „männlichen“ Idealtypus der Anwendung von Zeitmanagement bei Jugendlichen sprechen. Der weibliche Zeitmanagement-Typus zeichnet sich dabei dadurch aus, dass er zeitbezogene Volition als internalisierte Selbstverständlichkeit anwendet. Er benötigt deshalb kaum Willensanstrengung, um Handlungsabsichten aufrechtzuerhalten.

Vermeidungsstrategien werden abgelehnt und Prokrastination zur Entlastung genutzt, um erholt zu einem festgelegten Zeitpunkt mit der Handlung fortzufahren. Der „weibliche“ Idealtypus ist nicht offen für Ablenkungen und bewältigt gleichzeitig auftretende Handlungsnotwendigkeiten durch Multitasking. Der männliche Zeitmanagement-Typus übt zeitliche Volition als energieraubenden Selbstzwang aus. Gleichzeitig auftretende Handlungserfordernisse nutzt er zur Ablenkung, wobei oft ein Abbruch der ursprünglichen Handlungsabsicht folgt. Prokrastination wird ohne festen Zeitpunkt für die Fortführung der Handlung ausgeübt, auch hier droht der vollständige Handlungsabbruch. Diese Idealtypen dienen zur Beschreibung der Zeitmanagement-Realität von Jugendlichen, existieren aber in dieser „Reinform“ nicht, und es ist nicht erforscht, ob sie auch auf Erwachsene übertragbar sind.

Die Befragten mit Migrationshintergrund tendierten in dieser Kategorisierung zum „männlichen“ Zeitmanagement- Typen, obwohl dieser Teil der Untersuchungsgruppe mehrheitlich weiblich war.

Bei den männlichen Befragten ließ sich feststellen, dass ihr Zeitmanagement bei Vorhandensein einer Partnerbeziehung deutlich weiblicher wurde.

Die Befragten der Vergleichsgruppe der Auszubildenden zeigten einen stärker „weiblichen“ Zeitmanagement- Typus als die Teilnehmer der Berufsvorbereitung. Da diese Auszubildenden mehrheitlich am Anfang ihrer Ausbildung standen kann man davon ausgehen, dass sich der Zeitmanagement-Typus mit dem Erwerben des Status „Auszubildender“ änderte. Zeitmanagement lässt sich also nicht ausschließlich als erlernbare Fähigkeit betrachten, sondern es variiert mit dem aktuellen persönlichen Selbstkonzept, wie es sich durch das Erwartung-mal-WertModell beschreiben lässt.

Um Fähigkeiten des Zeitmanagements entwickeln zu können benötigen Jugendliche also nicht nur Lehr-Lern-Situationen, die Möglichkeiten zur zeitlichen 
Selbstregulierung eröffnen. Diese Situationen müssen auch in einen Kontext eingebunden sein, der realistische Zukunftsperspektiven eröffnet, damit volitionale Ressourcen aktiviert werden, die dem Zeitmanagement zugrunde liegen.

\section{Literatur}

Cavalli, A. 1988: Zeiterfahrungen von Jugendlichen. In: Zoll, R.: Zerstörung und Wiederaneignung von Zeit. Frankfurt am Main: Suhrkamp.

Dornbach, S. 2014: Zeitmanagement in der beruflichen Bildung. Wiesbaden: Springer VS.

König, C. J./Kleinmann, M. 2004: Zeitmanagement im Beruf: Typische Probleme und ihre Lösungsmöglichkeiten. In: Wiese, B. S. u. a.: Individuelle Steuerung beruflicher Entwicklung. Frankfurt am Main: Campus.

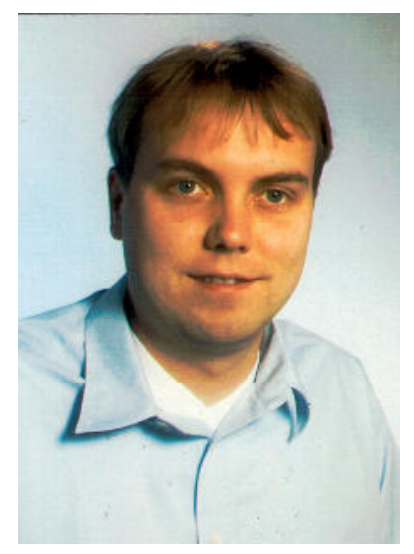

Dr. Stefan Dornbach

forscht zu Berufsausbildung in Brasilien und Deutschland. Postadresse: Frankfurter Allee 87, 10247 Berlin, E-Mail: dornbach@freenet.de 